\title{
Utentes sem equipa de saúde: uma prioridade e uma responsabilidade social
}

Paula Broeiro Gonçalves*

A criação do Serviço Nacional de Saúde (SNS) ${ }^{1}$ foi contemporânea e subsequente à Conferência de Alma-Ata, que veio evidenciar a saúde como um direito. A Declaração de Alma-Ata reconhece os cuidados primários de saúde como elemento-chave do desenvolvimento de justiça social e traçou, como objetivo, contrariar as desigualdades em saúde através de medidas políticas, económicas e sociais. ${ }^{2-3}$ Para se conseguir melhorar a saúde e bem-estar de todos e reduzir as desigualdades em saúde é preciso atingir dois objetivos políticos: criar uma sociedade que permita maximizar o potencial individual e da comunidade; e colocar no centro de todas as políticas a garantia de justiça social e de sustentabilidade nas decisões de saúde. ${ }^{4} \mathrm{O}$ reconhecimento das desigualdades sociais e da vulnerabilidade associada inspirou o desenvolvimento de sistemas de saúde de acesso universal (e.g., Português e Inglês), vindo ao encontro dos valores da saúde pública europeia: o bem comum e a responsabilidade coletiva. ${ }^{5}$

As desigualdades podem ser definidas como diferenças no estado de saúde ou na distribuição dos determinantes de saúde entre diferentes grupos populacionais, estando intimamente ligadas à privação socioeconómica (e.g., rendimento, habitação, trabalho, educação e saúde).$^{6}$ Conceito longe de ser consensual é, porém, um estado observável de desvantagem de cada indivíduo em relação à comunidade onde se insere. ${ }^{6}$

O índice de privação Europeu (EDI) é um índice ecológico, desenvolvido com base nas Estatísticas da União Europeia para o Rendimento e as Condições de Vida

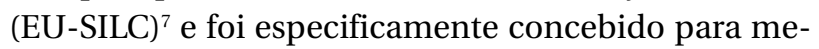
dir e monitorizar a pobreza e a privação em todo o ter-

* Médica de Família. UCSP dos Olivais, ACeS Lisboa Central.

Faculdade de Medicina da Universidade de Lisboa. ritório da União Europeia (UE) de forma a melhor entender desigualdades em saúde. ${ }^{6}$ Aplicando este EDI ao território continental português verifica-se um menor índice no litoral sul (Lisboa, Setúbal e Algarve), ${ }^{6}$ resultado coincidente com a distribuição geográfica da população estrangeira residente em Portugal (68\% registada nos distritos de Lisboa, Faro e Setúbal). ${ }^{8} \mathrm{O}$ fluxo migratório, ${ }^{8}$ após o declínio no período de austeridade, ${ }^{7}$ tem mantido uma tendência crescente evidenciada pelo crescimento do número de imigrantes da UE a residir em Portugal (cerca de $29 \%$ ), sendo os motivos mais relevantes na concessão de residência o reagrupamento familiar, a atividade profissional e o estudo. ${ }^{8}$ As nacionalidades predominantes são a brasileira, a italiana, a francesa e a britânica. ${ }^{8}$ No ACeS Lisboa Central os estrangeiros frequentadores provenientes da UE respeitam a hierarquia dos pedidos de residência no nosso país; dos oriundos de fora da UE destacam-se os brasileiros (8\%), logo seguidos dos $12 \%$ provenientes de países asiáticos (Nepal, Bangladesh, China e Índia).

O impacto da austeridade na saúde e nos cuidados de saúde foi estudado através das necessidades médicas não atendidas e seus motivos - antes, durante e após a recessão económica em Portugal. Verificou-se duplicação, entre 2010 e 2012, da possibilidade de ter tido uma necessidade médica não atendida $(\mathrm{OR}=2,41$; IC95\%, 2,01-2,89), ${ }^{7}$ com maior impacto nas pessoas com emprego. As razões para não procurar atendimento foram em $68 \%$ financeiras (OR=1,68; IC95\%, 1,32-2,12), o tempo de espera retirado ao trabalho ou a responsabilidades familiares (OR 2,18; IC95\%, 1,20-3,98). ${ }^{7} \mathrm{O}$ estudo ecológico realizado na UCSP dos Olivais mostrou resultados coerentes com aquele estudo - a população ativa (OR=1,2; IC95\%, $p<0,001)$ era o grupo com risco mais elevado de não ter médico de família (MF). ${ }^{9}$

No planeamento em saúde importa saber quem são os utentes SES inscritos nas Unidades de Cuidados de 
Saúde Personalizados (UCSP), de forma a melhor adequar os cuidados de saúde às necessidades da comunidade. Partilha-se, neste editorial, a experiência da UCSP dos Olivais, ACeS Lisboa Central, uma unidade funcional urbana litoral que reflete as carências de recursos humanos e materiais das UCSP e o padrão sociodemográfico da região em que se insere, como: o envelhecimento da população portuguesa ${ }^{11} \mathrm{e}$ os fluxos migratórios ${ }^{8,12}$ com expressão sociodemográfica. Os utentes SES estão inscritos como:

1. Sem MF atribuído - correspondendo a utentes com inscrição primária ativa (cidadãos de nacionalidade portuguesa e estrangeiros com residência em Portugal) por indisponibilidade de recursos de profissionais e de instalações;

2. Com inscrição esporádica-inclui utentes em mobilidade nacional [inscrição temporária prevista no Registo Nacional de Utentes (RNU)], com inscrição provisória (e.g., estrangeiros em regularização de residência) e os utilizadores do atendimento complementar (resposta do ACeS a doença aguda durante o fim de semana).

Excluindo este último, de resposta a doença aguda, reconhece-se a importância social do fenómeno «utentes SES»; no entanto, o conhecimento da sua magnitude não é possível por ausência de implementação da categorização prevista no RNU, publicado em agosto de $2017^{10}$ [inscrição ativa, inativa ou provisória; utentes com MF; sem MF por indisponibilidade para atribuição; ou sem MF por opção].

A equipa da UCSP dos Olivais, reconhecendo a vulnerabilidade da população SES, assume a responsabilidade coletiva e pratica solidariedade dando cumprimento aos valores éticos da saúde pública europeia, ${ }^{5}$ colaborando com o ACeS Lisboa Central, desde 2016, na gestão dos cuidados a esta população. Para tal, contratualizou horas médicas para cuidados a grupos com necessidades específicas (crianças até aos dois anos, grávidas, planeamento familiar e dependentes), bem como a renovação de receituário crónico, aplicando medidas de prescrição racional. Não se conhecendo o denominador por indisponibilidade dos dados do RNU quanto a inscrições temporárias e provisórias, constata-se que a contratualização de horas médicas é insuficiente para as necessidades e não se reflete no resultado da equipa cuja avaliação de desempenho é ex- clusivamente centrada nos atos e resultados prestados a utentes inscritos com médico de família. Acresce que cerca de $30 \%$ das horas de secretariado clínico e de cuidados de enfermagem são permanentemente alocadas ao atendimento e à prestação de cuidados à população SES, refletindo-se na sobrecarga destes profissionais, não reconhecida nem compensada pela tutela.

O Relatório de Primavera do Observatório Português dos Sistemas de Saúde (OPSS) de 2018, excluindo os utentes sem MF da análise, refere preocupação com a perpetuação das desigualdades no acesso aos CSP e com a prestação de cuidados de saúde em «dois níveis», ou seja, nas USF e UCSP. ${ }^{13}$ Esta assimetria não pode, no entanto, ser atribuída às UCSP mas ao desinvestimento neste modelo organizativo, a par da carência de profissionais no sul do país e ao crescimento de inscritos SES (inclui inscrições temporárias e provisórias).

O modelo organizativo USF, adequado a populações sociodemograficamente estáveis, tem trazido retorno de satisfação aos seus profissionais (autonomia de gestão e condições de exercício) e aos seus utilizadores. Contudo, revela-se como resposta insuficiente e inadequada a áreas metropolitanas que necessitam de respostas flexíveis, adaptáveis às mudanças sociodemográficas, como a mobilidade interna no país e os fluxos migratórios entre países.

A UCSP dos Olivais integra, na sua maioria, profissionais sem interesse pelo pagamento por desempenho, por escolha pessoal e ideológica. Tal como nas restantes UCSP são-lhe agregados os utentes SES que partilham dos seus recursos humanos (secretariado clínico e enfermagem) e materiais, já de si deficitários pelo desinvestimento da tutela neste modelo de cuidados holístico, biopsicossocial e centrado na pessoa e na comunidade. É, pois, necessário garantir a todas as unidades funcionais condições de exercício essenciais, de qualidade e segurança (estruturais, meios humanos, autonomia de gestão) e justas, de forma a responder às necessidades da população pela qual são responsáveis; é necessário repensar os cuidados aos utentes SES para não agravar as desigualdades em saúde, nem contaminar o desempenho das unidades funcionais, particularmente das UCSP. Subsistem, assim, críticas à evolução do modelo que mantém o foco no pagamento por desempenho ao: 
1. Perpetuar a competição que estimula o individualismo, ao invés de estimular a cooperação e o desenvolvimento de trabalho colaborativo entre unidades funcionais;

2. Normalizar os modelos organizativos, não dando espaço à diversidade e à flexibilidade que possam trazer igual retorno de ganhos em saúde e adaptar-se às necessidades específicas de cidadãos e profissionais.

A Administração Regional de Saúde de Lisboa e Vale do Tejo, além de ser uma região carente de profissionais de saúde, é ainda a que recebe o maior número de pessoas por mobilidade interna ${ }^{12}$ e por resultado dos fluxos de imigração. ${ }^{8}$ Ao invés de facilitar a evolução quase coerciva para o modelo USF, deveria incentivar modelos criativos que se adequem à comunidade que servem e às mudanças sociodemográficas, reconhecendo-os como um contributo para a minimização das desigualdades em saúde.

\section{REFERÊNCIAS BIBLIOGRÁFICAS}

1. Lei n 56/79, de 15 de setembro. Diário da República. 1979;। Série(214).

2. World Health Organization. Declaration of Alma-Ata: international conference on primary health care, Alma-Ata, USSR, 6-12 September 1978 [Internet]. Geneva:WHO; 1978.Available from: https://www.who. int/publications/almaata_declaration_en.pdf

3. Brito-de-Sá A. De Alma-Ata a Astana: o futuro, hoje [From Alma-Ata to Astana: the future, now]. Rev Port Med Geral Fam. 2019;35(2):80-1. Portuguese

4. Caan W. Fair society, healthy lives: timing is everything. BMJ. 2010;340: c1191.

5. Camps V, Hernández-Aguado I, Puyol A, Segura A. An ethics training specific for European public health. Public Health Rev. 2015;36:6.

6. Ribeiro Al, Mayer A, Miranda HP, Pina MF. A versão Portuguesa do European Deprivation Index: um instrumento para o estudo das desi- gualdades em saúde [The Portuguese version of the European Deprivation Index: an instrument to study health inequalities]. Acta Med Port. 2017;30(1):17-25. Portuguese

7. Legido-Quigley H, Karanikolos M, Hernandez-Plaza S, Freitas C, Bernardo L, Padilla B, et al. Effects of the financial crisis and Troika austerity measures on health and health care access in Portugal. Health Policy. 2016;120(7):833-9.

8. Ribeiro M], editor. Relatório de imigração, fronteiras e asilo, 2017 [Internet]. Barcarena: Serviço de Estrangeiros e Fronteiras; 2017. Available from: https://sefstat.sef.pt/Docs/Rifa2017.pdf

9. Cardoso S, Broeiro P. Associação entre marcadores de vulnerabilidade social e atribuição de médico de família num centro de saúde: estudo ecossocial [Internet]. In: $21^{\circ}$ Congresso de Medicina Geral e Familiar, Vila Real, 29 de setembro a 1 de outubro de 2017. Available from: https://prezi.com/p/jg_m-iz9tqje/associacao-entre-marcadoresde-vulnerabilidade-social-e-atribuicao-de-medico-de-familia-numcentro-de-saude-estudo-ecossocial/

10. Asministração Central do Sistema de Saúde. Regulamento do registo nacional de utentes (RNU) [Internet]. Lisboa: ACSS; 2017. Available from: http://www.acss.min-saude.pt/wp-content/uploads/2017/08/ Regulamento-Registo-Nac.-Utentes.pdf

11. Oliveira CR, Rosa MS, Mota-Pinto A, Botelho AS, Morais A, Verissimo MT. Estudo do perfil do envelhecimento da população Portuguesa [Internet]. Coimbra: Universidade de Coimbra; 2010. ISBN 978-989-844500-1. Available from: http://rihuc.huc.min-saude.pt/handle/10400.4/ 992

12. D'Uva TB, Fernandes M. Mobilidade social em Portugal [Internet]. Lisboa: Fundação Francisco Manuel dos Santos; 2017. Available from: https://www.ffms.pt/FileDownload/19af23f9-53ca-477b-9ac75949df7f79ef/mobilidade-social-em-portugal

13. Observatório Português dos Sistemas de Saúde. Meio caminho andado: relatório Primavera 2018 [Internet]. Lisboa: OPSS; 2018. ISBN 978972-97492-2-3. Available from: http://opss.pt/wp-content/uploads/ 2018/06/relatorio-primavera-2018.pdf

\section{ENDEREÇO PARA CORRESPONDÊNCIA}

E-mail: paulabroeirorpmgf@gmail.com https://orcid.org/0000-0002-5013-6171 